\title{
High Spatial/Energy Resolution Band Gap Measurements: Delocalization and Other Effects in a Monochromated Cold FEG Nion Dedicated STEM.
}

\author{
R. W. Carpenter ${ }^{1,3}$, H. Xie ${ }^{2,4}$ T. Aoki ${ }^{3}$, and F. A. Ponce ${ }^{4,3}$.
}

${ }^{1}$ Dept. of Chemistry and Biochemistry, Arizona State University, Tempe, USA.

${ }^{2}$ School for Engr. of Matter, Transport, and Energy, Arizona State University, Tempe, USA.

${ }^{3}$ LeRoy Eyring Center for Solid State Science, Arizona State University, Tempe, USA.

${ }^{4}$ Dept. of Physics, Arizona State University, Tempe, USA.

Measuring band gaps and delocalization with a STEM microscope requires a small probe, for spatial resolution, and high enough energy resolution and stability to both resolve the gap and minimize the energy width of the ZLP so that the gap structure is not obscured. The Nion microscope used here is located in a specially prepared very stable site and has demonstrated $20 \mathrm{meV}$ energy resolution with a 2 $\AA$ probe, and small enough ZLP base width to observe small band gaps and lattice phonon losses $[1,2,3]$. The ratio of Nion to typical Schottky FEG TEM/STEM ZLP width at 1\% of maximum intensity is 0.035 . This ratio will vary a bit depending on how current is adjusted in the probe, but in general it is small, due the monochromator and cold FEG. Here we show results for band gap measurements relative to edge-on interface position in two cross section specimens. In one specimen the materials on opposite sides of the interface have quite different gaps $\left(\mathrm{Al}_{2} \mathrm{O}_{3}, \mathrm{E}_{\mathrm{g}} \sim 9 \mathrm{eV}\right.$ and $\left.\mathrm{GaN}, \mathrm{E}_{\mathrm{g}} \sim 3.4 \mathrm{eV}\right)$ and in the other the difference is small ( $\mathrm{AlGaN}$ multiquantum well superlattice, $\mathrm{E}_{\mathrm{g}} \sim 5 \mathrm{eV}$ and $\mathrm{AlN}, \mathrm{E}_{\mathrm{g}} \sim 6.2 \mathrm{eV}$ ). The main results for both materials systems were the same: when the probe was on the interface the band gaps for both materials in the couple were visible in the spectrum, and when the probe was moved away from the interface the band gap signal of the material containing the probe remained strong, while the gap signal from the material on the opposite side of the interface deceased until it disappeared when the probe was sufficiently far from the interface.

Some of the results for the $\mathrm{Al}_{2} \mathrm{O}_{3} / \mathrm{GaN}$ interface are shown in Fig. 1. The distance from the interface where the $\mathrm{GaN}$ gap signals disappears is $\sim 62 \AA$, and the distance for $\mathrm{Al}_{2} \mathrm{O}_{3}$ gap signal disappearance is $\sim$ $39 \AA$. Similar results are shown for the MQW superlattice/GaN specimen in Fig. 2. Here, the MQW gap signal disappears about $67 \AA$ from the interface on the AlN side, but on the MQW side the gap is still between 5 and $6 \mathrm{eV}$ at $17 \AA$ from the interface, i.e. $17 \AA$ is not far enough from the interface for the effect of the larger AlN gap to disappear. In this latter case the gap difference is only about $1 \mathrm{eV}$, and it was difficult to resolve the two gaps simultaneously because their onsets are not sharp. The magnitude of the delocalization lengths we observed are in remarkably good agreement with the approximate values given by Egerton for losses smaller than $50 \mathrm{eV}: \sim 35 \AA$ for $10 \mathrm{eV}$ loss and $\sim 60 \AA$ for $5 \mathrm{eV}$ loss[4]. The shapes of the loss curves of Figs. 1 and 2 introduce some uncertainties into band gap measurements, especially the higher energy band gap of each pair, because the loss curve for that constituent lies on top of the lower gap loss curve, making background correction difficult. This effect can increase the apparent gap by a small amount. Nevertheless our values for AlN and GaN measured away from interfaces are in reasonably good agreement with reported values [5]. A wide range of values have been reported for $\mathrm{Al}_{2} \mathrm{O}_{3}$, with the highest values near about $9 \mathrm{eV}$, and lower values reported for specimens containing impurities or exhibiting Urbach tails [6]. During optical band gap measurements for $\mathrm{Al}_{2} \mathrm{O}_{3}$ Tomiki et. al. observed a significant peak at $\sim 6 \mathrm{eV}$ loss that they attributed to impurities [6], which is similar to the broad peak also centered at $\sim 6 \mathrm{eV}$ that we observed for the $\mathrm{Al}_{2} \mathrm{O}_{3}$ loss curve (upper curve of Fig. 1). We also attribute this midgap feature in $\mathrm{Al}_{2} \mathrm{O}_{3}$ spectra to impurities. Additional data for other systems will also be presented. 


\section{References}

[1] RW Carpenter et al, Micros. Microanal. 18(Supp 2) (2012), p.408.

[2] O L Krivanek et al, Microscopy 62(1) (2013), p.3.

[3] O L Krivanek et al, Nature 514 (2014), p.209.

[4] R F Egerton, Ultramic. 107 (2007) p.575.

[5] S Bloom, J Phys Chem Solids 32 (1971) p.2027.

[6] T Tomiki et al, Jour Phys Soc (Japan) 62(No. 3) (1993) p.573.
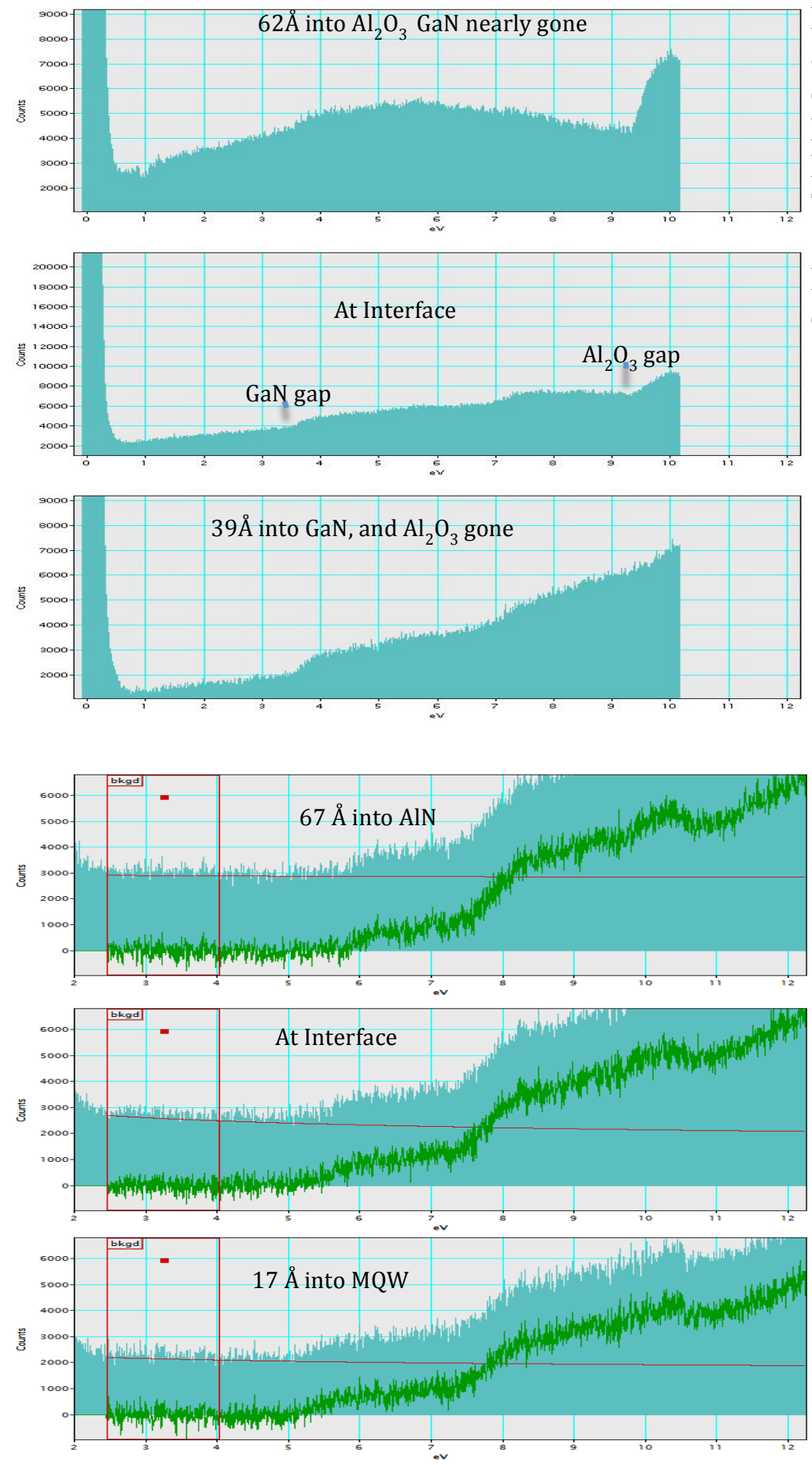

Figure 1. Three low loss spectra from an $\mathrm{Al}_{2} \mathrm{O}_{3} / \mathrm{GaN}$ interface specimen. Top curve, $62 \AA$ into $\mathrm{Al}_{2} \mathrm{O}_{3}$ from interface. Middle curve, probe on interface, Bottom curve, probe $39 \AA$ into $\mathrm{GaN}$. These spectra are from a line scan of 100 steps, of $2.4 \AA$ length.

Recorded at $60 \mathrm{kV}, 0.005 \mathrm{eV} / \mathrm{ch}$ dispersion. Probe size $\sim 2 \AA$. 